\title{
Costes y Beneficios Sociales de la Enseñanza Universitaria a Distancia.
}

\author{
(Socio-Economics of the Virtual University) \\ CHRIS CURRAN \\ Dublin City University \\ (Irlanda)
}

\begin{abstract}
RESUMEN: La mayoría de las investigaciones y publicaciones sobre costes de la educación a distancia en la universidad se han centrado en los costes soportados por las instituciones que ofertan este tipo de programas. De hecho muchas de las investigaciones se han centrado en un tópico interesante, pero relativamente reducido: la unidad de coste soportado por las universidades abiertas frente a la unidad de coste de las universidades tradicionales del mismo país. Dada la importante misión social de la mayoría de las universidades abiertas (y muchas a distancia), sorprendentemente se ha prestado poca atención al concepto más amplio de costes y beneficios sociales de la universidad a distancia. Este artículo toca algunos de estos temas: la demanda social de educación a distancia, el apoyo estatal, los costes comparativos, quién paga, los beneficios sociales y particulares de la participación en la educación a distancia, el papel de la educación a distancia en el desarrollo del capital humano, y la 'misión social' de esta modalidad educativa. Este artículo pretende servir para estimular el interés por el análisis de los costes y beneficios sociales y, en particular, fomentar la recopilación de datos transnacionales que apoyen una análisis comparativo profundo y más comprehensivo.
\end{abstract}

Costes de la Educación a Distancia - Costes y Beneficios Sociales - Educación Universitaria

\begin{abstract}
Most research and publication on the costs of distance education at University level have focussed on the costs incurred by the institutions providing programmes. Indeed much of this research has centred on an interesting, but relatively narrow, topic - the unit costs incurred by open universities relative to those of traditional universities in the same country. Given the stated social mission of most open (and many distance teaching) universities, surprisingly little attention has been directed to the wider social costs and benefits of university distance teaching. This paper raises a number of these issues: the social demand for distance education; state support; comparative cost; who pays?; the private and social benefits of participation in distance education; the role of distance education in developing human capital; and 'social mission' of this mode of education. It is hoped that the paper will serve to stimulate interest in the analysis of social costs and benefits and in particular encourage the compilation of transnational data to support a more comprehensive and in-depth comparative analysis.
\end{abstract}

Costs of Distance Education - Social Costs and Benefits - University Level

\footnotetext{
* Este artículo será publicado en breve en inglés como parte del volumen titulado "Experiences from Open and Distance Teaching Universities in Europe (A Speculation by Distance Educators)". Deutscher Studien-Verlag (DSV), Weinheim, Alemania, 2000.
} 


\section{INTRODUCCIÓN}

MacArthur ${ }^{1}$ presenta una breve pero muy interesante interpretación de la fase de gestación de la propuesta para crear una Universidad Abierta en el Reino Unido. En dicha presentación detalla la evolución de la propuesta desde que fue inicialmente concebida por el entonces primer ministro Harold Wilson el Domingo de Resurreción de 1963, continuando con el nombramiento de Jennie Lee como ministro responsable de la misma, la designación de una comisión asesora y más tarde de un comité de planificación constituido por eminentes académicos bajo la presidencia de Sir Peter Venables, la publicación de un Libro Blanco y las complejas y a menudo intensas negociaciones con varios ministerios, la BBC y otras instituciones implicadas. A pesar de este largo proceso de consultas, negociaciones y planificación, el resultado final se mantuvo incierto hasta el final

Me sería muy grato poder decir que la Universidad Abierta... representó el triunfo de una idea irresistible a la que le había llegado su momento. Pero no fue así. En cualquier momento, hasta que los primeros alumnos no comenzaron el curso en Enero de 1971, podía haber sido reprimida por hombres sin ninguna imaginación; y estuvo a punto de ocurrir así.

Visto desde la perspectiva de hace treinta años, esta incertidumbre parece extraña, por no decir inexplicable. La Universidad Abierta del Reino Unido, con más de 100.000 alumnos, es considerada por la mayoría como un evidente éxito. Hoy en día, en casi todos los países desarrollados, la enseñanza a distancia se considera parte integrante del sector de la enseñanza superior. Los gobiernos de la mayoría de los países ofrecen algún tipo de financiación pública para sufragar la enseñanza a distancia. ¿Por qué había de ser difícil conseguir el consenso necesario en una socialdemocracia moderna entre los políticos e instancias del poder?

Una de las respuestas podría ser que la enseñanza a distancia, así como otras formas de enseñanza superior, no es más que uno de los muchos objetivos sociales deseables a los que los gobiernos podrían asignar los escasos recursos de los que disponen. Desde esta perspectiva económica, la pregunta podría reformularse de una forma ligeramente distinta. ¿Por qué querrían los gobiernos crear una universidad abierta, o financiar la enseñanza a distancia? ¿Por qué los escasos recursos públicos habrían de destinarse a esta actividad y no a otras valiosas iniciativas? Teniendo en cuenta que aquellos que participan en la enseñanza a distancia obtienen considerables beneficios por hacerlo, ¿por qué debería la sociedad apoyar dicha participación?

Es importante destacar que los costes sociales de la enseñanza a distancia son afrontados por la sociedad en su totalidad, no sólo por las universidades que ofrecen los programas. La totalidad de estos costes sociales comprenden aportaciones directas tales como los gastos administrativos y las tasas académicas, lo que viene a ser el coste de los recursos utilizados junto con el valor de cualquier pérdida de bienestar de terceras partes, una consideración que se hace más obvia en áreas como la contaminación ambiental que en educación. ¿Por qué debería la sociedad soportar esta carga? Concretamente, ¿por qué los gobiernos deberían invertir los pocos recursos a su disposición en financiar la enseñanza a distancia, o incluso, la enseñanza superior en general?

\subsection{RAZONES ECONÓMICAS}

Uno de los argumentos clave a favor de dicho apoyo reside en las consecuencias externas que se derivan de la educación, es decir, en unos beneficios que van más allá de los alumnos y licenciados directamente implicados y que repercuten en la sociedad en general. Es ampliamente reconocido que la sociedad se beneficia de la educación de los individuos que la componen y que estos "beneficios sociales" se añaden a los "beneficios particulares" que genera en los alumnos, que son el objeto de la educación. En consecuencia, se argumenta que el nivel de la demanda privada, si se deja a elección de los individuos, sería demasiado bajo (considerando la sociedad en su totalidad). Para compensar esta deficiencia la sociedad necesita subvencionar la oferta educativa ${ }^{2}$.

Muchos de los beneficios de la educación están directamente relacionados con objetivos sociales clave; por ejemplo, el desarrollo de las aptitudes y la preparación necesaria para el funcionamiento de una economía moderna. Como refiere Schultz,

Sencillamente, no es posible sacar partido de una agricultura moderna y una industria próspera sin realizar importantes inversiones en los seres humanos ${ }^{3}$.

Esta idea, la de que la educación representa un medio eficaz de generar capital (en forma de capacidad y preparación humana más que de equipamientos o instalaciones) no es nueva. En efecto, el concepto de invertir en capital humano es una idea bastante recurrente en la literatura económica ${ }^{4}$. desde que Adam Smith, en su obra La riqueza de las naciones, comparara a un hombre bien preparado con una costosa máquina ${ }^{5}$. Sin embargo, no fue hasta los años sesenta cuando 
esta idea se expresó claramente en una teoría coherente y estructurada. Denison, en su tan influyente análisis sobre el crecimiento económico en los Estados Unidos, definía las mejoras en educación como un factor principal de crecimiento económico ${ }^{6}$ y la inversión en capital humano como la explicación fundamental al "inexplicable" aumento de la producción nacional. Este punto de vista, común a muchas otras teorías económicas, no dejó de cuestionarse ${ }^{7}$. en su momento. No obstante, las primeras investigaciones empíricas ya demostraban que los beneficios de la inversión en educación eran superiores a los de la inversión en capital físico; más tarde, exhaustivas investigaciones arrojaron resultados situados típicamente en un tramo del 5 al $15 \%$. Estudios posteriores basados en datos longitudinales también apoyaron la teoría de que (manteniéndose iguales otras condiciones) los trabajadores con una formación superior disfrutaban de mejores salarios que aquellos de nivel educativo más bajo. Consecuentemente, la creación de capital humano ha llegado a ser considerada como una tarea fundamental de la educación y, por tanto, de la educación superior.

\subsection{POLÍTICA SOCIAL Y EDUCACIÓN A DISTANCIA}

Pero, ¿es la educación a distancia un medio eficaz para el desarrollo de capital humano? ¿Genera los mismos beneficios que otros modos de enseñanza tradicional? Incluso si damos por válida la argumentación del apoyo social, quedan por contestar muchos interrogantes referidos al aspecto de la política social.

¿Qué alcance debería tener el apoyo estatal a la educación a distancia en términos absolutos y en relación con otras áreas de la enseñanza superior (o incluso a otras áreas de política social tales como la atención sanitaria o la reducción de la criminalidad)? ¿Quién debería costearla? ¿Debería la enseñanza a distancia ser gratuita? ¿Tendrían los alumnos que pagar la totalidad o parte de las tasas o habría que ofrecerles préstamos a devolver después de la obtención del título? ¿Quién debería beneficiarse de la educación a distancia? ¿Debería la financiación pública ofrecerse a los más brillantes (que pueden sacar más partido de la enseñanza, aprender más rápido y quizá rentabilizar más su aprendizaje, sirviendo así más eficazmente a los intereses de la sociedad en general)? ¿O más bien debería dirigirse a los educativamente desaventajados (que requerirían una financiación per cápita más costosa para alcanzar cierto grado de igualdad de oportunidades)? ¿Habría que financiar con más generosidad ciertos tipos de enseñanza a distancia (como los programas dirigidos a paliar las deficiencias respecto a las capacidades de los individuos más que los de filosofía, humanidades o cursos culturales)? ¿Qué aspectos de la enseñanza a distancia directamente relacionados con los intereses de la sociedad (si es que pueden identificarse) "justificarían” un mayor respaldo? ¿Tendría que ser el sistema de enseñanza a distancia en general lo más homogéneo posible, dando a acceso a instituciones en gran medida equivalentes y con titulaciones comunes? ¿O sería mejor que fuese un sistema diversificado para acomodarse a las demandas de poblaciones estudiantiles dispares? ¿Tendría que haber un único proveedor central de enseñanza a distancia, o debería promoverse la participación de muchas universidades?

Este tipo de interrogantes son los que se plantean cuando los responsables políticos deciden crear una universidad de enseñanza a distancia y también (aunque de forma menos evidente) cuando las medidas políticas, las prácticas y los programas de las universidades de enseñanza a distancia cambian o evolucionan con el paso del tiempo.

¿Cómo responder a este tipo de preguntas? En principio, la respuesta dependería de las prioridades sociales. En la práctica, las decisiones son generalmente el resultado un a menudo complejo proceso de negociaciones en las que participan los responsables políticos, los ministerios que desempeñan alguna función dentro del área (por ejemplo, los ministerios de educación y economía) y los responsables de crear y dirigir las universidades de enseñanza a distancia. Como se afirmaba en la versión de MacArthur sobre la evolución de la propuesta para crear la Universidad Abierta del Reino Unido, este proceso puede ser largo, complejo y los resultados inciertos.

Las decisiones que se tomen pueden considerarse como la expresión pública de la política social. ¿Cómo han encontrado expresión estas decisiones (respecto a la enseñanza a distancia) en otros países de la UE? ¿Han sido concordantes en los distintos países o existen notables diferencias entre unos y otros? Para abordar estas cuestiones tenemos que referirnos directamente a la enseñanza universitaria a distancia en Europa, pero primero deberíamos considerar brevemente el alcance y la importancia de esta actividad en los países de la UE.

\section{LA INSCRIPCIÓN EN LA ENSEÑANZA A DISTANCIA}

La demanda global de la enseñanza a distancia está conformada por la suma de las demandas particulares de muchos individuos. Por este motivo se ve en gran medida influida por factores de tipo estrictamente personal tales como clase, renta, entorno familiar, sexo o intereses culturales y especialmente por las oportunidades de avance profesional y social que la participación en la enseñanza superior ofrece a los individuos. Dados los importantes beneficios derivados de la participación en la enseñanza superior y los cuantiosos subsidios que recibe en la mayoría de los países reduciendo así el coste privado de la misma, no es de extrañar la fuerte demanda de que es objeto la enseñanza superior. 
En muchos países (especialmente de los países en vías de desarrollo, donde la tasa de beneficios puede ser el doble que la de los países desarrollados) la demanda de enseñanza superior puede llegar a exceder con creces la oferta disponible. En otros países la oferta y la demanda pueden acercarse más a un punto de equilibrio. En Estados Unidos, por ejemplo, donde existe un nivel de participación muy alto entre la población en edad universitaria y un desarrollado sistema de acceso para los adultos a la enseñanza superior, la mayoría de las personas que quieren ingresar en la enseñanza superior probablemente pueden hacerlo. No obstante aquí la competición por entrar en las instituciones más prestigiosas es muy intensa por lo que el exceso de demanda es de carácter estratificado y no global.

\subsection{LA DEMANDA DE ENSEÑANZA A DISTANCIA}

La enseñanza a distancia tiene sus orígenes en la década de $1830^{9}$; durante unas cuantas décadas este tipo de programas se ofrecieron con normalidad y en algunos casos extensivamente en países como el Reino Unido, Alemania, Estados Unidos y Japón ${ }^{10}$. Ello se debió en gran parte a la iniciativa de personas particulares y empresas privadas que demandaban una mejor educación para los individuos, con el fin de aprovechar las oportunidades de progreso derivadas del desarrollo industrial así como de la expansión del sector público. La participación directa de la universidad en esta primera fase de la enseñanza a distancia fue, sin embargo, bastante vacilante. No obstante, se produjeron algunas iniciativas por parte de las universidades ${ }^{11}$ y hacia el final del siglo ya se había creado el primer departamento de enseñanza por correspondencia en la Universidad de Chicago. En Australia la Universidad de Queensland creó un Departamento de Estudios Externos en $1911^{12}$, al principio sólo para un puñado de estudiantes.

El crecimiento fue considerable durante las décadas siguientes. Especialmente en Estados Unidos ${ }^{13}$, Canadá ${ }^{14}$, Australia $^{15}$ y la Unión Soviética, donde la enseñanza a distancia fue adoptada a finales de los años veinte para mejorar los resultados del sistema educativo ${ }^{16}$, y en la antigua República Democrática Alemana, donde aproximadamente un cuarto de los licenciados por la universidad y las escuelas técnicas

“...obtenían sus títulos a través de la enseñanza a distancia."."17.

Debería quedar claro, por tanto, que antes incluso del nacimiento de las universidades abiertas, la enseñanza a distancia constituía ya un sector muy importante de la enseñanza superior en algunos países.

No obstante, el establecimiento de la Universidad Abierta en el Reino Unido (UKOU*.) en $1969^{18}$. marcó un avance significativo. El enfoque adoptado por la UKOU era muy innovador por centrarse exclusivamente en la enseñanza a distancia, por el alto grado de compromiso con el objetivo de ampliar el acceso de los adultos en edad madura a las carreras universitarias, por su planteamiento innovador y multimediático de la enseñanza y su característico aperturismo. La Universidad tuvo un impacto inmediato en la ampliación del acceso a la enseñanza superior dentro del Reino Unido, con una inscripción inicial de 19.581 alumnos en 1971, que dos décadas más tarde se cuadriplicó (alcanzando la cifra de $81.575^{19}$. alumnos). Dentro del extenso mundo de la enseñanza a distancia este desarrollo ha tenido una gran influencia. Durante las dos décadas siguientes se crearon otras universidades abiertas: cuatro ${ }^{20} \cdot$ de ellas $^{2}$ en Europa y más de veinte en otros países del mundo.

\subsection{LA INSCRIPCIÓN EN LA ENSEÑANZA A DISTANCIA EN LOS PAÍSES DE LA UE}

La educación a distancia es actualmente una parte muy importante del sector de la enseñanza superior de los países de la UE, con un exceso total inscripciones que afecta aproximadamente a medio millón de alumnos. Lamentablemente los datos sobre las cifras de inscripción en los distintos países son parciales e incompletos. No obstante, las universidades abiertas establecidas en cinco países de la UE son los principales proveedores de enseñanza a distancia y la inscripción en estas instituciones representa un indicador aproximado del alcance que tiene la enseñanza a distancia en estos países. En el caso del Reino Unido, por ejemplo, había 189.000 alumnos matriculados en la Open University para el curso 1995-96. Esta cifra representa aproximadamente el 84 por ciento de la inscripción total en los programas de enseñanza a distancia de las instituciones de enseñanza superior ${ }^{21}$. En el caso de Alemania, la FernUniversität era la única universidad de la antigua RFA que ofrecía enseñanza a distancia. Algunas universidades de la antigua RDA sí lo hacían aunque no se tiene conocimiento del cuál era el alcance de su implicación en este tipo de enseñanza. En segundo lugar, el gobierno federal hasta hace poco tiempo no había ofrecido ningún tipo de incentivos para promover la introducción de nuevas tecnologías en las universidades, lo que hará que de ahora en adelante otras universidades aparte de la FernUnversität se vean motivadas para iniciar su actividad en este campo. Teniendo en cuenta todo esto, los datos sobre las inscripciones en las universidades abiertas representan una útil (si bien incompleta) estimación del alcance y la importancia de la enseñanza a distancia en los cinco países.

\footnotetext{
*. UKOU: United Kingdom Open University (N. del T.)
} 
Tabla 1: La inscripción en la enseñanza superior y la inscripción por créditos en las Universidades Abiertas de cinco países de la UE.

\begin{tabular}{|l|l|l|}
\hline País & $\begin{array}{l}\text { Inscripción en la Enseñanza } \\
\text { Superior (000) }\end{array}$ & $\begin{array}{l}\text { Inscripción por créditos en las } \\
\text { Universidades Abiertas (000) }\end{array}$ \\
\hline Alemania & $2.155,7$ & 46,5 \\
\hline Holanda & 502,9 & 29,0 \\
\hline Portugal & 300,6 & 5,2 \\
\hline España & $1.527,0$ & 138,7 \\
\hline Reino Unido & $1.813,3$ & 117,1 \\
\hline
\end{tabular}

Fuente $^{22}$ : año 1994-95

Fuente $^{23}$ : año 1995

En la Tabla 1 se muestra la inscripción en la enseñanza superior en cinco países de la UE que cuentan con Universidades Abiertas, así como las cifras correspondientes a la inscripción en los programas por créditos de dichas instituciones. Como se puede observar, la importancia de la enseñanza a distancia con respecto a los datos de la inscripción total en la enseñanza superior varía de un país a otro, desde el 2,2\% en Alemania al 10\% en España. No obstante, en ningún caso es menospreciable. Diez países de la Unión Europea no cuentan con universidades abiertas y aunque los datos correspondientes a estos países son menos fiables, sí pueden ofrecer alguna información. Se sabe por ejemplo que en Francia, dentro del nivel universitario, unos 36.000 alumnos se habían matriculado en los cursos por créditos de la FIED y unos 5.000 en los programas de la CNED. Si se contempla desde una perspectiva global, parece razonable concluir que la universidad a distancia representa un sector muy significativo de la enseñanza superior en los países de la Unión Europea.

\section{APOYO ESTATAL A LA ENSEÑANZA A DISTANCIA}

El gasto público en enseñanza es una de las formas de medir el compromiso social y representa una parte importante del producto nacional bruto en la mayoría de los países desarrollados, donde normalmente se sitúa en torno a un 5-6\%. La enseñanza superior viene a constituir una quinta parte de ese gasto ${ }^{24}$, alrededor del $1 \%$ del producto nacional bruto, si bien la proporción varía notablemente de un país a otro (desde un $14 \%$ en Francia hasta un $32 \%$ en Holanda ${ }^{25}$ ) en la unión Europea ${ }^{26}$.

La información sobre el nivel de gasto público en el sector de la enseñanza a distancia no es homogénea entre los diversos países de la Unión Europea y resulta incompleta en algunos países concretos. Sí se disponen de algunos datos sobre la financiación estatal de las universidades abiertas. El presupuesto de la FernUniversität en 1998, por ejemplo, fue de 125 millones de marcos alemanes ${ }^{27}$, constituyendo la asignación más importante de fondos del estado dentro del marco de la enseñanza a distancia, pero no incluye el gasto en otras universidades ni en la Fachoschule. La Open University en el Reino Unido (UKOU) recibió 119 millones de libras esterlinas ${ }^{28}$. para la enseñanza y la investigación en 1996/97; este dato de nuevo representa la asignación de recursos públicos más cuantiosa dentro del área de la enseñanza superior en el Reino Unido, pero no incluye el gasto efectuado en otras treinta universidades de enseñanza a distancia (gran parte de él subsumido en los presupuestos generales). Los datos sobre el gasto público en diez países de la Unión Europea son todavía más limitados ${ }^{29}$.

Aunque no es posible facilitar una estimación completa del gasto público de la enseñanza a distancia en los países de la UE, al menos sí es posible ofrecer una estimación aproximada de los cinco países que cuentan con universidades abiertas. La tabla 1 presenta los datos sobre el gasto público en la enseñanza superior y de las subvenciones estatales a las universidades abiertas por MECU de GDP. Dado que los datos se refieren sólo a universidades abiertas éstos resultan incompletos y, por tanto, la estimación es inferior a la real respecto al volumen total del gasto público en la enseñanza a distancia en estos países. No obstante, dado que estas universidades abiertas reciben la mayor parte de la financiación pública destinada a la enseñanza a distancia en los respectivos países, la subestimación es probablemente poco significativa. 
Tabla 2: Gasto público en la enseñanza superior y subvenciones estatales a las universidades abiertas (por 100.000 ECU de GDP) en cinco países de la Unión Europea.

\begin{tabular}{|l|l|l|}
\hline País & $\begin{array}{l}\text { Gasto en enseñanza superior } \\
\text { ECU por GDP (MECU) }\end{array}$ & $\begin{array}{l}\text { Subvención estatal a la } \\
\text { Universidad Abierta } \\
\text { ECU por GDP (MECU) }\end{array}$ \\
\hline Alemania & 9.765 & 31 \\
\hline Holanda & 14.569 & 119 \\
\hline Portugal & 8.191 & 108 \\
\hline España & 6.779 & 70 \\
\hline Reino Unido & 12.600 & 164 \\
\hline
\end{tabular}

Fuente $^{30}$ : año 1995

Fuente $^{31}$ : año 1995

Como se puede observar, las subvenciones estatales a las universidades abiertas y consecuentemente el gasto público en la enseñanza a distancia representa una parte significativa aunque relativamente pequeña de los recursos destinados a la enseñanza superior, variando desde el $0,4 \%$ de Alemania al 1,3\% del Reino Unido.

La cuestión que puede resultar más interesante sería comparar lo que el estado se gasta por estudiante en la enseñanza a distancia con otras formas más tradicionales de enseñanza superior. Existen pruebas que apuntan a que dicho gasto (coste público) puede ser menor en la enseñanza a distancia. Horlock ${ }^{32}$, en un estudio sobre la Open University del Reino Unido, comparaba el gasto público realizado en 1982 por cada licenciado en Humanidades de dicha universidad con el de otras universidades convencionales del Reino Unido. Los resultados sugerían que los gastos de la UKOU representaban aproximadamente el $61 \%$ del de las universidades convencionales del Reino Unido. La composición de los costes podía variar ligeramente dado que se centraba en los costes públicos y excluía por tanto las tasas de tutoría y las becas de mantenimiento para los estudiantes de la UKOU, aunque las incluía en los costes de las universidades convencionales basándose en que en este último caso sí eran pagadas con fondos públicos.

Si relacionamos la información de las Tablas 1 y 2 es posible comparar el gasto público medio por inscripción de la enseñanza superior en general, por un lado, y de las universidades abiertas por otro. En todos los casos el gasto de la primera supera al de la segunda en una proporción de 5 a 1 en el caso del Reino Unido y de 9 a 1 en el de España. El escaso gasto público per cápita o por inscripción que se aplica a toda la enseñanza superior en general es una característica común (aunque no universal) de la enseñanza a distancia, lo que en realidad equivale a decir que cada vez más personas tienen acceso a la enseñanza superior (a través de la enseñanza a distancia) sin que haya aumentado el gasto público.

Lo anterior no significa que el coste medio por alumno equivalente sea necesariamente más bajo, sino simplemente que el coste de la matrícula o inscripción es menor. ¿Por qué hacemos esta distinción? La gran mayoría de los alumnos en la enseñanza a distancia estudian con dedicación parcial y, por tanto, los datos sobre las necesidades de inscripción deberían ser corregidas por un factor desconocido (que a menudo se calcula en un 0.5 aproximadamente) para garantizar que la comparación se basa en estudiantes con dedicación exclusiva (FTEs) ${ }^{1}$. Por otra parte, el número de alumnos actualmente matriculados en los programas de enseñanza a distancia no es necesariamente el mismo que el de los alumnos activos. Dada la naturaleza de la enseñanza a distancia, la proporción de alumnos inactivos tiende a ser más alta que en la enseñanza tradicional. Una de las universidades abiertas facilitó unos datos donde se sugiere que el $48 \%$ de la población actualmente matriculada estaba inactiva. Por tanto, en el presente, deberíamos darnos por satisfechos con la perspectiva de que el coste de matrícula o inscripción en la enseñanza a distancia pueda ser relativamente bajo, como efectivamente es el caso.

\section{COSTE COMPARATIVO}

¿Por qué se compara el coste medio por alumno con el de otras formas de enseñanza? Es ampliamente compartido que la enseñanza a distancia representa una alternativa de bajo coste a otras formas tradicionales de enseñanza superior (de hecho esta teórica ventaja se aduce a menudo para justificar que la financiación pública sea más baja) La mayoría de las investigaciones empíricas sobre la economía de la enseñanza a distancia se ha centrado en un aspecto relativamente limitado: ¿cuál es el resultado de la comparación entre los costes unitarios por estudiante de enseñanza a distancia con los de la enseñanza universitaria con dedicación exclusiva dentro de un mismo país? Algunos estudios han arrojado

\footnotetext{
${ }^{1}$. Full Time Students (N. del T.)
} 
resultados altamente positivos, indicando unos costes unitarios que representan una cuarta parte de los de otros cursos similares de la enseñanza con dedicación exclusiva. Los resultados, sin embargo, han sido generalmente menos favorables en la enseñanza a distancia, donde la comparación se establece con la enseñanza con dedicación parcial (y no con dedicación exclusiva), donde se tienen en cuenta otras actividades como la investigación y donde los licenciados, o los créditos conseguidos, constituyen la base de la comparación y no simplemente el número de alumnos.

Condicionamientos de este tipo, así como el relativamente escaso número de universidades de enseñanza a distancia que han sido objeto de una investigación externa y metodológicamente fiable, debería inclinarnos a ser cautos. Quizá lo más que podríamos decir es que, dadas las circunstancias adecuadas, la enseñanza a distancia puede representar una alternativa económicamente eficaz a modos más tradicionales de enseñanza universitaria.

Que en la práctica éste llegue a ser el caso depende de una serie de factores, especialmente de las alternativas disponibles para alcanzar los objetivos educativos deseables y de las estrategias de funcionamiento adoptadas por las universidades de enseñanza a distancia con respecto sobre todo al desarrollo y entrega de los cursos. La enseñanza a distancia constituye un sector diferenciado, pero, contra la creencia popularmente extendida, en ningún caso homogéneo, de la enseñanza superior, y la adopción de estrategias inadecuadas a su escala de funcionamiento puede traducirse en unos costes más altos y no más bajos por estudiante o por licenciado (respecto a sistemas más tradicionales de enseñanza y aprendizaje). No obstante, en aquellos casos en los que la enseñanza a distancia ha sido correctamente diseñada y resulta adecuada al volumen de inscripción, se pueden economizar los costes operativos.

Existen numerosas pruebas que demuestran que la enseñanza a distancia puede proporcionar un ahorro sustancial en el capital destinado a infraestructuras. En un reciente estudio sobre la UKOU, Wagner estimaba que la inversión de capital por alumno equivalía sólo al 6\% del de las universidades convencionales, incluso si se excluían los costes residenciales de las universidades convencionales ${ }^{33}$. La mayoría de las comparaciones de esta clase se hacen, sin embargo, con programas con dedicación exclusiva tradicionales y por ello tienen más relevancia en aquellos países donde la enseñanza a distancia representa una alternativa a la enseñanza con dedicación exclusiva. Sin embargo, la enseñanza a distancia en Europa se ofrece normalmente a alumnos adultos que estudian con dedicación parcial, los cuales, aunque se inscribieran en otra modalidad educativa que no fuera la enseñanza a distancia, requerirían una menor provisión de capital para infraestructuras. A pesar de ello, incluso en este caso se podría conseguir un importante ahorro en determinadas situaciones.

\section{5. ¿QUIÉN PAGA LA ENSEÑANZA A DISTANCIA?}

Un bajo nivel de financiación estatal no significa necesariamente que la enseñanza a distancia resulte más económica. Puede significar que algunos de los costes hayan sido transferidos de los fondos públicos a otras instancias. Ello nos lleva a considerar cómo se distribuyen los costes de la enseñanza a distancia o quién paga la enseñanza a distancia.

Podríamos comenzar por afirmar que la financiación del gobierno es sólo una fuente de financiación y que la importancia de esta fuente de financiación varía considerablemente entre los distintos países de la Unión Europea. La financiación del gobierno expresada como porcentaje de los ingresos totales se sitúo en torno al 35\% en el caso del Centro Nacional de Enseñanza a Distancia de Irlanda, un 44\% en el caso de la UNED, un 57\% en el caso de la Open University del Reino Unido, un $73 \%$ en el de la Open University de Holanda y un casi $100 \%$ en el de la FernUniversität ${ }^{34}$. En la mayoría de los casos el déficit (entre la financiación estatal y los ingresos totales) es asumido principalmente por los ingresos procedentes de las tasas, de modo que la responsabilidad de financiar la enseñanza a distancia recae, en mayor o menor grado, sobre los estudiantes, sus familias o sus empresas.

\subsection{EL GASTO FAMILIAR}

La mayoría de las modalidades de enseñanza superior acarrean algún tipo de gasto para los alumnos o sus patrocinadores. El gasto directo de las familias en la enseñanza superior en forma de tasas o materiales de estudio y los gastos indirectos derivados de dicha inscripción en la enseñanza superior (traslados, alojamiento, manutención etc.) puede ser considerable, pero varía sustancialmente de un país a otro. Las tasas de matrícula en Europa son relativamente bajas si se comparan con las de Japón o Estados Unidos y el gasto privado es generalmente más bajo en la UE que en muchos otros países, especialmente del mundo en desarrollo ${ }^{35}$. Las universidades de los países de la UE reciben gran parte de su financiación del estado (si se compara por ejemplo con Estados Unidos y Japón, donde la proporción de los ingresos procedentes de las tasas es sustancialmente superior a los procedentes del erario público).

En aquellos casos en que la financiación estatal es relativamente baja, las tasas de los alumnos a menudo representan la principal fuente alternativa de ingresos, por lo que la política en cuanto al pago de las tasas y la cantidad a pagar también varían notablemente entre las distintas universidades de enseñanza a distancia. Los alumnos de la FernUniversität no pagan tasas (aunque se les puede cobrar una pequeña cantidad por materiales de enseñanza). En 
cambio, los alumnos del Centro Nacional de Educación a Distancia de Irlanda (NDEC) pagan tasas bastante cuantiosas (del orden de los 6.000 ECU por programa de licenciatura ${ }^{36}$.

\subsection{BECAS}

La carga de las tasas a ciertos alumnos puede verse aligerada mediante las becas, sean de la índole que sean. En general, los alumnos de la enseñanza a distancia no reciben becas de mantenimiento por parte del estado, lo que sí ocurre en el caso de los alumnos a tiempo completo. ${ }^{37}$, pero sí pueden, sin embargo, recibir becas por el coste de la enseñanza. Kirkwood refiere que en 1986 aproximadamente el 12\% de los estudiantes de la UKOU solicitaron a las autoridades locales una beca de este tipo, y alrededor del $6 \%$ recibieron ayudas financieras totales o parciales ${ }^{38}$. Otro $29 \%$ recibió ayuda de su empresa o de la Universidad (5\%). Por otro lado, al 38\% de los alumnos que realizaron cursos semanales en escuelas de verano se les subvencionó totalmente el pago de las tasas y un $24 \%$ recibió ayudas parciales. Las empresas pueden constituir una fuente importante de financiación, especialmente en aquellos casos en los que los cursos guardan relación con el tipo de trabajo que está desempeñando el alumno dentro de la empresa. Un estudio sobre los alumnos del NDEC (de un curso de Tecnología de la Información) mostraba que alrededor de un 50\% de las tasas las pagaban íntegramente sus empresas y a otro $20 \%$ se les sufragaban en parte. El apoyo de los empresarios a los alumnos inscritos en un tipo de cursos menos directamente relacionados con el trabajo (por ejemplo, Humanidades) es significativamente menor.

\subsection{PRÉSTAMOS}

Algunas universidades ofrecen acceso a sus alumnos a planes de préstamos (especialmente para la compra de equipamiento), a veces a través de acuerdos establecidos con agentes financieros externos. La Universitat Oberta de Catalunya incorpora un campus virtual, por lo que es necesario que los alumnos tengan acceso a ordenadores y otros equipamientos (cuyo coste se estimó en 1.350 ECU en 1997). Para que los alumnos puedan comprar el equipamiento necesario, la universidad ha previsto un plan de préstamos "de bajo coste" a través de las Cajas de Ahorros y la Cámara de Comercio ${ }^{39}$.

En general los alumnos de la enseñanza a distancia normalmente no tienen que pagar tanto dinero como sus compañeros de otros tipos de enseñanza por los gastos indirectos (traslados, alojamiento), ya que estos son menores al no tener las mismas obligaciones respecto a la asistencia a clase.

\subsection{PRIVACIÓN DE INGRESOS}

La privación de ingresos puede representar un coste de oportunidad muy significativo para los alumnos con dedicación exclusiva de las universidades convencionales. Asimismo, la pérdida de producción implica un coste social importante: una estimación realizada sugiere que la privación de ingresos, en cuanto a porcentaje sobre el coste social total de cada alumno es del orden del $50 \%$ en los países desarrollados ${ }^{40}$.

Los alumnos de enseñanza a distancia gozan de ventaja a este respecto, dado que no necesitan dejar su empleo para cursar sus estudios, por lo que continúan contribuyendo a la economía nacional. Esta es una fuente importante de ahorro. Sin embargo, la mayoría de las comparaciones de esta índole parten de la base de que los estudiantes a tiempo completo no realizan trabajos remunerados, lo que (según sugiere la observación de la realidad) actualmente no es cierto en la mayoría de los casos (al menos en muchos países europeos). Más importante aún es la suposición implícita de que el tiempo libre que los alumnos invierten en la enseñanza a distancia no tiene ningún valor. Mientras los estudios comparativos sigan sin tener en cuenta estas consideraciones, los potenciales ahorros, por más que reales, corren el peligro de sobreestimarse.

\subsection{FINANCIACIÓN PRIVADA}

El sector privado (por ejemplo fundaciones, empresas e individuos) es otra fuente de financiación de las universidades. La información fiable respecto de la financiación privada de la enseñanza superior es bastante limitada, pero no obstante se cree que es poco significativa si se compara con el gasto público en la mayoría de los países de la UE. (Los Estados Unidos podrían representar la excepción en este aspecto; una estimación sugiere que en 1985-86 las aportaciones privadas, becas y contrataciones ascendieron al 5,4\% y los ingresos por donaciones al $2,3 \%$ de los fondos actuales de las instituciones americanas de enseñanza superior ${ }^{41}$ ).

Todavía más desconocido es el alcance de la financiación privada en la enseñanza a distancia. Puede suponerse sin embargo que, en Europa, la financiación procedente de donaciones es aún menor que la de las universidades convencionales (aproximándose a cero). Pocas o quizá ninguna universidad a distancia (puede que debido al hecho de su relativamente reciente creación) han creado una asociación de antiguos alumnos a la que poder recurrir en busca de 
apoyo financiero. La financiación de la industria para apoyar el desarrollo de algunos programas podría constituir un recurso potencialmente viable; de hecho algunas, si bien pocas, universidades de enseñanza a distancia ya han establecido relaciones con empresas privadas, autoridades locales u organismos semi-estatales para los cuales han encargado o diseñado especialmente cursos por una cantidad previamente negociada.

Queda claro que la parte más importante de la financiación de la enseñanza a distancia en estos países procede del estado, pero que hay otras fuentes, siendo los alumnos, sus familias o las empresas donde trabajan importantes colaboradores. El alto grado de variación respecto a la financiación del gobierno en los distintos casos sugiere que la percepción oficial con respecto al papel de la enseñanza a distancia como instrumento de política social es muy variada.

\section{BENEFICIOS DE LA ENSEÑANZA SUPERIOR A DISTANCIA}

Si partimos de la base de que la renta ingresada por los individuos se aproxima al valor marginal de su contribución a la sociedad, la renta adicional ingresada por los individuos puede interpretarse como una medida del valor social de la educación. Existe abundante literatura científica que demuestra que la participación en la enseñanza superior genera un rendimiento privado positivo a los individuos, en la forma de futura fuente de (mayores) ingresos. Las estimaciones varían de unos casos a otros, pero un rendimiento promediado del 12\% parece una estimación razonable si nos referimos a la enseñanza superior en los países desarrollados ${ }^{42}$. Una vez más, sin embargo, se producen considerables variaciones entre los distintos países; una publicación reciente registra el índice interno del rendimiento económico que supone la enseñanza universitaria en diecisiete países de la OCDE; los índices en los casos de las mujeres en Alemania, Holanda, el Reino Unido y Portugal son del 8,2 \%, 10,5\%, 19,1\% y 28,3\% respectivamente ${ }^{43}$.

La investigación sobre el rendimiento privado de los licenciados en la enseñanza a distancia tiene un alcance muy limitado, a pesar de lo cual se han manifestado algunas dudas respecto a los beneficios económicos que pueden obtener de su educación ${ }^{44}$, en comparación con los licenciados de la enseñanza superior con dedicación exclusiva. Mace planteó la cuestión de si los beneficios económicos derivados de una licenciatura en la UKOU pudiesen ser tan altos como los de un título obtenido en cualquier universidad convencional del Reino Unido aunque sólo sea porque a la edad de treinta y siete años,

“...la mayoría de las personas ya tienen un trabajo establecido y, además, existen poderosas fuerzas de tipo institucional, tales como los mercados internos de trabajo, que impedirían su movilidad." 45.

La observación de una serie de universidades de enseñanza a distancia de Europa sugiere que este punto de vista es injustificadamente pesimista, al menos con respecto a sectores concretos del mercado laboral. La experiencia irlandesa demuestra que los licenciados en algunos programas universitarios tales como Tecnología de la Información, Enfermería y algunas áreas de Empresariales parecen tener poca dificultad para avanzar en su profesión mediante la movilidad laboral (un estudio sobre los licenciados en Tecnología de la Información mostraba que el 70\% había cambiado de empleo durante el periodo de realización de sus estudios o posteriormente ${ }^{46}$ ). Esto parece cumplirse también en el caso de los "mejores" licenciados en carreras de carácter más general, si bien el proceso puede requerir un periodo intermedio que se invierte en la obtención de mejores cualificaciones (por ejemplo, trabajos de investigación para obtener un master o un doctorado).

La experiencia irlandesa parece repetirse en otros países de Europa. En un estudio realizado con 4.500 licenciados por la UKOU, el 57\% expresó que su rendimiento laboral o perspectivas profesionales habían mejorado como consecuencia de sus estudios, y el 74\% dijo haber experimentado ya algún tipo de mejora laboral ${ }^{47}$, de los cuales el $53 \%$ declaraba haberlo conseguido con la ayuda de su titulación en la Open University. De manera que más de un tercio de los licenciados creían al menos que sus titulaciones en la UKOU les habían beneficiado ${ }^{48}$. Otro estudio similar realizado con estudiantes de la FernUniversität ${ }^{49}$. mostró que un $41 \%$ de los alumnos consideraban que habían obtenido beneficios de sus estudios en cuanto a la posibilidad de cambiar de trabajo. Otro estudio posterior arrojó resultados todavía más positivos, ya que los autores expresaron que aquellos licenciados que habían tenido empleo de forma continuada y que actualmente ocupaban un trabajo retribuido con dedicación exclusiva, o que trabajaban como autónomos,

habían duplicado sus salarios desde el momento en que se habían inscrito en la enseñanza a distancia hasta el momento actual. ${ }^{50}$.

Asimismo un estudio realizado con ex-alumnos de un programa en Suecia, llevado a cabo cinco años después de haber finalizado dicho programa, demostró que la situación laboral del 46\% de estos ex-alumnos había cambiado de una forma u otra, y de ellos la mitad habían cambiado de empleo. ${ }^{51}$. 
Parecen existir pocas dudas de que los licenciados en programas de enseñanza a distancia de estas universidades europeas perciben su participación en ellos como valiosa. Sin embargo, esta evidencia, aunque llamativa, no constituye por si sola suficiente base para comparar la rentabilidad de la enseñanza a distancia con la de la enseñanza convencional. En primer lugar, las respuestas son esencialmente cualitativas (ninguno de los estudios se ha propuesto calcular la tasa de rentabilidad de la participación en la enseñanza a distancia. En segundo, no podemos estar seguros de hasta qué punto los estudios a distancia son responsables de los efectos positivos sobre el empleo. Las personas que han completado con éxito un exigente programa universitario a la vez que ocupaban un puesto de trabajo a tiempo completo, y aunque no tengamos en cuenta las responsabilidades domésticas y de otro tipo propias de la edad adulta, a menudo presentan un excepcional nivel de motivación y compromiso, seguramente mayor al que se requiere para participar en la enseñanza superior a la edad convencional. Además, muchos ya tienen alguna experiencia en un determinado campo que está relacionado con sus estudios. Estas son características que podrían contribuir al éxito.

Por otra parte, Muta y Saito presentan evidencias ${ }^{52}$ que pueden entrar en conflicto con las opiniones positivas expresadas anteriormente. Los autores realizaron una encuesta por correo con los licenciados por la Universidad del Aire de Japón (UAJ) en la que se incluían preguntas sobre el efecto económico de su aprendizaje en la UAJ ${ }^{53}$. Los resultados mostraron un efecto positivo en el caso de los trabajadores que tenían la misma formación (que la de los estudiantes de la UAJ) antes de su matriculación. El aumento del "valor económico" se situaba entre el 2 y el $14 \%$ dependiendo del nivel académico anterior a su matriculación. Los resultados fueron menos positivos cuando se compararon con los de los licenciados por las universidades convencionales. El "valor económico" en el caso de los licenciados por la UAJ fue aproximadamente un $8 \%$ más bajo. Lamentablemente, al no haber estudios similares sobre otras universidades de enseñanza a distancia no es posible evaluar hasta qué punto este modelo se cumple en la enseñanza a distancia en general.

\subsection{LA ENSEÑANZA A DISTANCIA Y LA CREACIÓN DE CAPITAL HUMANO}

Este estudio plantea una cuestión interesante. Desde la perspectiva de la rentabilidad social, ¿genera la enseñanza a distancia el mismo nivel de capital humano que la enseñanza tradicional? Sorprendentemente, existen pocos estudios referidos a esta cuestión, pero hay razones para preocuparse. La edad es una de ellas. La edad de los licenciados (de la enseñanza a distancia) varía según los programas, pero los 35 años podrían tomarse como una edad bastante representativa. Los alumnos de la enseñanza tradicional se licencian a una edad mucho más temprana. Aunque esta edad varía entre los distintos países, los veintitrés años podrían tomarse en este caso como la más representativa. Si fijamos los sesenta y cinco como la edad de jubilación, está claro que los beneficios para la sociedad son menores, alrededor de un setenta por ciento $(71.4 \%)$ de la rentabilidad en la enseñanza tradicional.

Gran parte de la rentabilidad derivada del aumento de la inversión en capital humano, sin embargo, sólo se consigue muchos años más tarde de haber realizado la inversión. Descontar esta futura rentabilidad del valor actual reduciría el diferencial. Además, cuando se tiene en consideración el efecto de no tener que renunciar al trabajo mientras se está estudiando, el "valor actual" de la alternativa de la enseñanza a distancia es más alto y no más bajo que el de la alternativa tradicional. A modo de ejemplo...

\subsection{EQUIVALENCIA DE RESULTADOS}

Incluso si los resultados de las universidades a distancia (en forma de alumnos o licenciados) tuvieran un coste igual o incluso menor (que el de las universidades tradicionales), ¿serían equivalentes? ¿Es la enseñanza a distancia tan útil como los modos tradicionales de enseñanza?

Esta pregunta no es nueva. Los educadores a distancia (especialmente, quizás, los pioneros de las universidades abiertas) están demasiado familiarizados con el escepticismo e incluso la hostilidad con que se reciben sus propuestas para crear universidades de enseñanza a distancia ${ }^{54}$. Las críticas más repetidas ${ }^{55}$. son "no funcionará", "tiene menos nivel que los programas universitarios tradicionales", "la instrucción es demasiado directiva" o "los estudiantes tienen escaso nivel académico". La mayoría de estos temores hace tiempo que se olvidaron y son pocos actualmente los que cuestionan la capacidad de los sistemas de enseñanza a distancia bien diseñados y dotados de los recursos necesarios para impartir una materia académica de gran calidad y apoyar a los alumnos en su aprendizaje. La UKOU, por ejemplo, ocupó recientemente el décimo lugar entre 120 universidades del Reino Unido, dada la calidad de su enseñanza (y se sitúo por delante de las universidades de Oxford, Cambridge y la London University es cuanto a la calidad de su enseñanza en las carreras de ingeniería).

No existen razones, en principio, por las que el nivel de una licenciatura obtenida mediante la enseñanza a distancia sea inferior al de cualquier otra conseguida mediante la asistencia a un campus. Ello es especialmente cierto, quizás, en países (como Estados Unidos, Australia y Canadá) donde la enseñanza a distancia está firmemente integrada en el sistema de enseñanza tradicional y donde la mayoría de las universidades de prestigio imparten un programa de 
licenciatura, en el campus y a distancia, que es similar en todos los aspectos, excepto en el modo de transmisión. En general podría decirse que los beneficios derivados del contenido de la enseñanza a distancia no deberían, en principio, ser distintos de los de la enseñanza tradicional; la diferencia, si es que la hay, reside en los respectivos procesos.

\subsection{LA EXPERIENCIA UNIVERSITARIA}

La enseñanza eficaz no es más que una parte de lo constituye una "buena" enseñanza universitaria. Gran parte del aprendizaje universitario, se argumenta, se produce a través del debate con otros estudiantes, o con los profesores fuera de las clases, y esta interacción social se considera importante a la hora de estimular la curiosidad intelectual, la creatividad académica y la autoconfianza ${ }^{56}$ del alumno. Los defensores de esta forma de interacción social perciben la universidad como el "lugar" por antonomasia, en palabras de Newman". Otros pueden argumentar que las universidades son esencialmente comunidades ${ }^{58}$. y que con los avances de la tecnología moderna, el lugar ya no es esencial para la interacción social. Por otra parte, este tipo de críticas subestiman sin duda la importante fuente de comunicación que en muchas, sino en la mayoría de las universidades, representan las tutorías, las escuelas de verano y de fin de semana o las tutorías por medios informáticos y audiovisuales. De hecho, a los educadores a distancia a veces les parece que su forma de enseñanza se compara, no con la enseñanza convencional que se practica normalmente en las universidades tradicionales, sino con una especie de ideal, que, si es que existió alguna vez, en la actualidad desde luego no prolifera.

\subsection{BENEFICIOS NO-MONETARIOS}

Los beneficios de la enseñanza superior no se limitan a los efectos que puede generar en los ingresos. Existen otros beneficios adicionales, no monetarios, que los estudiantes obtienen a partir de su participación en la enseñanza superior. Estos rendimientos no materiales, si bien menos tangibles que un aumento de los ingresos, pueden ser no menos valiosos. Entre ellos se incluye por ejemplo la mejora del status, el acceso a puestos de trabajo más interesantes o deseables y (al menos en algunos casos) una capacidad para apreciar "las cosas importantes" de la vida". Muchos estudiantes también extraen un beneficio significativo de su participación en la enseñanza superior, que para unos puede radicar en la oportunidad de satisfacer sus intereses en determinados ámbitos académicos, para otros reside en el desafío intelectual que representa una "buena" enseñanza universitaria y para muchos en tener ocasión de encontrarse con otros jóvenes y relacionarse socialmente con sus iguales en un ambiente relativamente relajado y atractivo durante un periodo de unos tres o cuatro años.

¿Hasta qué punto la enseñanza a distancia produce beneficios no monetarios de este tipo?

\subsection{BENEFICIOS EXTERNOS INDIRECTOS}

Es ampliamente compartido que los beneficios externos menos directos de la enseñanza son también sustanciales ${ }^{60}$. Algunos de los beneficios sociales y políticos más importantes incluyen por ejemplo la facilitación del funcionamiento democrático a través de un electorado mejor informado, la prevención del crimen por medio del refuerzo de comportamientos socialmente aceptables (especialmente entre los jóvenes) o la creación de una sociedad dispuesta a acometer los cambios necesarios. Este tipo de beneficios, aunque reales, son en todo caso difíciles de cuantificar.

No obstante, en principio no habría razón por la que la enseñanza a distancia hubiera de ser menos eficaz en este sentido que otras formas más tradicionales de enseñanza, hecha la salvedad de que la mayoría de los estudiantes, al ser de edad más madura, probablemente ya han adquirido las convenciones y actitudes propias de su edad.

\section{LA ENSEÑANZA A DISTANCIA Y LA POLÍTICA SOCIAL}

La mayoría de estas cuestiones, a las que ya nos referíamos al comienzo de este documento, son objeto de continua investigación por parte de economistas y otros profesionales de las ciencias sociales relacionados con la educación en general, por lo que existe abundante literatura sobre el tema. Prácticamente todas estas cuestiones (y otras relacionadas con ellas) vienen son objeto de discusión (a veces muy intensa) para políticos y responsables educativos. Los estudios comparativos sobre los enfoques que aplica cada país a la facilitación y financiación de la enseñanza superior sugieren que las medidas adoptadas por los gobiernos (si bien tienen mucho en común) se aplican muy diferentemente en la práctica. Existen notables diferencias entre Estados Unidos y Europa, por ejemplo, respecto a la diversidad de estructuras y fuentes de financiación, así como en cuanto a la estructura de los cursos o la importancia de la jerarquía dentro de los departamentos académicos, por nombrar sólo algunas. ${ }^{61}$. Incluso dentro de Europa, se pueden encontrar sorprendentes diferencias en cuanto a las tendencias de matriculación ${ }^{62}$ o la distribución de los alumnos entre los distintos campos de estudio. 


\subsection{FUNCIÓN SOCIAL DE LA ENSEÑANZA A DISTANCIA}

¿Desempeña la enseñanza a distancia una función social especial? Las cinco universidades abiertas que funcionan en Europa han sido creadas por sus respectivos gobiernos ${ }^{63}$ y a todas ellas se les ha conferido la autoridad y los recursos necesarios para llevar a cabo su función dentro de la enseñanza superior. En otras universidades (que funcionan de manera dual) la enseñanza a distancia es una más de sus actividades, para lo cual reciben financiación del gobierno, ya sea de forma directa mediante un presupuesto especial, o indirecta, como parte de la financiación global de la universidad.

Como ocurre en el caso de sus equivalentes tradicionales, las universidades de enseñanza a distancia cumplen múltiples objetivos. Sin embargo, la mayoría de las universidades de enseñanza a distancia creadas a finales de los años sesenta, setenta y ochenta, tenían en común el desempeño de una misma función, la de compensar las desigualdades en la distribución del acceso a la enseñanza superior, ofreciendo una alternativa y un sistema de amplia disponibilidad para acceder a los estudios universitarios. ¿Hasta qué punto estas instituciones han cumplido con este objetivo?

El desarrollo de unos sistemas eficaces y ampliamente accesibles indudablemente se ha conseguido. El éxito a la hora de compensar las desigualdades ha sido en cambio más discutible. Son relativamente pocos los alumnos de programas de enseñanza a distancia que proceden de los sectores culturalmente más bajos de la sociedad. El porcentaje de alumnos matriculados en la UKOU que no presentaban cualificaciones oficiales antes de su ingreso fue de menos del cinco por ciento en 1971 y permanecía por debajo del diez por ciento en 1991 ${ }^{64}$. El porcentaje de alumnos de la Universidad Abierta de Holanda que no contaban más que con estudios de primaria o una formación profesional básica, era sólo del $3 \%{ }^{65}$. en 1994 . Incluso las universidades de enseñanza a distancia con fórmulas de libre entrada no han conseguido atraer a gran número de estudiantes educativamente desaventajados ${ }^{66}$, o ni siquiera lo han intentado con el debido empeño ${ }^{67}$. Ello no es sorprendente si tenemos en cuenta la insistencia en establecer estándares académicos completamente equivalentes a los de las universidades tradicionales y la evidente correlación empírica entre el bajo nivel educativo anterior a la matriculación en la enseñanza a distancia y los altos índices de fracaso académico. Muchos estudiantes de programas enseñanza a distancia tienen ya alguna modalidad de titulación de tipo terciario (certificado, diploma o graduado); no obstante, muchos educadores de instituciones enseñanza a distancia podrían citar importantes excepciones al respecto ${ }^{68}$ y el porcentaje de alumnos que no cumplen con los requisitos normales de matriculación es normalmente más alto que en las universidades tradicionales.

\subsection{POBLACIONES OBJETIVO}

No sería justo evaluar la enseñanza a distancia desde la única perspectiva de su éxito a la hora de reducir las desigualdades educativas. La mayoría de las universidades a distancia desempeñan actualmente un papel muy significativo en la mejora de las capacidades y los conocimientos de los individuos, que a menudo ya cuentan con unas buenas cualificaciones y están activos en el mundo laboral. Estos estudiantes a menudo se matriculan en una sola asignatura o como mucho en unas cuantas, en lugar de hacerlo en un programa universitario completo. Un excesivo y exclusivo interés en facilitar el acceso a titulaciones universitarias a los desaventajados conduce a infravalorar la importancia de estas otras inscripciones, llegando al extremo de considerarlas como abandonos académicos prematuros $^{69}$. En efecto, la acogida de estos estudiantes bien cualificados constituye un medio importante para promover el aprendizaje permanente para un importante sector de la economía. Los beneficios sociales podrían ampliarse más allá de la simple mejora de las aptitudes y los conocimientos de los individuos. La mejora de la preparación de los profesionales o directivos altamente cualificados puede tener un efecto positivo y muy valioso en la productividad de los trabajadores que están a su cargo.

Sin embargo, estos alumnos son claramente diferentes de los individuos educativamente desaventajados (que normalmente son considerados el objetivo fundamental de la enseñanza a distancia) ya que con frecuencia pertenecen a sectores económicamente favorecidos de la sociedad $\mathrm{y}$, además, esperan beneficiarse personal, $\mathrm{y}$ a veces sustancialmente, de su participación en estos programas. Desde la perspectiva de la política social, ¿sería adecuado asignar menos recursos a estos alumnos que ya están titulados y que han recibido importantes subvenciones estatales para su educación, sobre todo si se hace a expensas de otros individuos más desaventajados? En este mismo sentido, muchos alumnos que están matriculados en universidades tradicionales, se inscriben en programas de enseñanza a distancia aunque solo sea por acceder a los materiales de los cursos, que son de una alta calidad académica y pedagógica. ¿Deberían estos estudiantes recibir dos subvenciones estatales?

La respuesta adecuada dependerá en principio de las prioridades sociales y educativas; en la práctica, por supuesto, dependerá en gran parte del contexto. Los programas desarrollados para mejorar las capacidades de los profesores contratados directamente por el estado, sobre todo si se ha diseñado con el fin de facilitar la aplicación determinadas medidas gubernamentales (como los cambios curriculares), probablemente recibirán una consideración distinta a los 
programas ofrecidos a directivos o contables de la banca privada (aun cuando su contribución a la sociedad pueda ser igualmente importante).

Preguntas de índole similar pueden plantearse cuando se insta (ya sea el gobierno o sus agentes) a las universidades de enseñanza a distancia a desempeñar una función precursora respecto a la utilización de nuevas tecnologías en la enseñanza universitaria. El uso eficaz de las nuevas tecnologías en la educación puede generar unos beneficios sociales al garantizar una importante reducción del coste educativo, al mismo tiempo que mantiene o aumenta el nivel de calidad. Los programas de este tipo son a menudo objeto de iniciativas de financiación especiales por parte de los gobiernos.

\section{CONCLUSIONES}

Es evidente que la enseñanza superior absorbe una importante cantidad de los escasos recursos de los que dispone la sociedad. El gasto público en la enseñanza superior es del orden del 1\% en los países de la UE y a ello debería añadirse el gasto privado (que es bastante bajo en los países de la UE), el gasto familiar y el coste derivado de la privación de ingresos.

La información sobre el gasto en la enseñanza a distancia es escasa. Al menos en algunos países, el coste público per cápita es bajo en relación con el de la enseñanza convencional con dedicación exclusiva, especialmente en lo referente a las infraestructuras. El gasto público general en becas es bajo en relación con el de los alumnos con dedicación exclusiva y por esta sola razón el coste público por estudiante tiende a ser más bajo en la enseñanza a distancia que en la enseñanza con dedicación exclusiva. Esta financiación más baja se compensa no obstante por el incremento de las tasas de los alumnos. El efecto que esto supone respecto a la diversidad de programas que se ofrecen no se ha determinado, pero probablemente esté lejos de ser neutral. En igualdad de circunstancias las instituciones que necesitan recaudar importantes ingresos en concepto de tasas probablemente optaran por programas que sean comercialmente viables y dirigidos a poblaciones estudiantiles que pueden pagar, o conseguir que les paguen, las tasas requeridas.

Resulta evidente que la enseñanza a distancia ha conseguido con éxito ampliar el acceso a la universidad en los países de la UE. Incluso la más fría de las valoraciones reconoce que la enseñanza a distancia ha facilitado el acceso de muchos miles de alumnos y que el volumen de inscripción representa hoy en día una parte importante de la población universitaria de dichos países. La ampliación de la oferta de programas que se ha ido produciendo con el paso del tiempo y los tipos de población estudiantil a los que se ha dirigido ha reforzado la tendencia hacia un mayor crecimiento. No obstante, el éxito a la hora de atraer a los individuos educativamente desaventajados ha sido bastante limitado. En efecto, podría argumentarse que el aprendizaje continuado y permanente son las áreas en las que la enseñanza a distancia ha producido mayor impacto y que representan la fuente más importante de beneficios sociales.

El importante crecimiento que protagonizado la enseñanza a distancia durante las últimas décadas sugiere que la enseñanza a distancia goza hoy en día de una posición relativamente alta. Ello no fue siempre así, es más, en sus comienzos, las universidades a distancia se toparon con una fuerte oposición. Lord Perry, fundador y vicerrector de la British Open University, señala que, al principio, el sistema iniciado por su universidad fue recibido por la mayoría de los académicos con gran desconfianza. En su opinión,

Se tardó cinco años... en alcanzar una respetabilidad académica como sistema complementario de enseñanza superior $^{70}$.

No obstante, las universidades abiertas tuvieron gran importancia a la hora de elevar el prestigio de la enseñanza a distancia, rescatándola de la marginalidad y situándola como parte integrante de la enseñanza superior.

El éxito de la enseñanza a distancia es indudablemente la principal razón que ha motivado la mejora de su posición. Este éxito se evidencia no sólo en lo referente a la enseñanza a distancia o en la calidad académica o instructiva de muchos de los cursos que se ofrecen, sino también en la reconocida satisfacción de los alumnos con la experiencia de la enseñanza a distancia y con los beneficios que ha generado para sus licenciados posteriormente ${ }^{71}$, aunque la evidencia de esta conexión es todavía algo escasa. También se evidencia en la aceptación por parte de las universidades tradicionales de las innovaciones introducidas o que al menos se han hecho más visibles en las universidades de enseñanza a distancia. Dichas innovaciones son de diversa naturaleza y algunas revisten más importancia que otras, pero incluso en una breve enumeración de las mismas deberá incluirse la creciente imbricación de las universidades tradicionales en la enseñanza a distancia, el énfasis actual en el desarrollo de las capacidades de los alumnos que asisten al campus como estudiantes autónomos, la estructura cada vez más modular de los cursos y la posibilidad de acumulación de créditos, así como la marcada tendencia a la matriculación con dedicación parcial por parte de los alumnos no tradicionales de edad madura en los programas de asistencia al campus. Más reveladora todavía sea quizá la 
voluntad de las universidades tradicionales de aceptar a los licenciados en programas de enseñanza a distancia como estudiantes postgraduados y de investigación, y en casos excepcionales, como titulares de sus puestos académicos.

Sin embargo, la amplia comunidad académica de la mayoría de los países percibe una dicotomía entre la enseñanza a distancia y la enseñanza tradicional por la cual las diferencias son mayores que las similitudes. La mayoría de los alumnos a distancia son de edad madura, es decir, mayores que los que van a la universidad, y a menudo no se les exige que aprueben un examen de ingreso antes de ser admitidos para estudiar cualquiera de las carreras universitarias. En consecuencia, los alumnos de los programas de enseñanza a distancia son generalmente clasificados como "no tradicionales", "externos", "extramuros" o con alguna otra designación que, aunque cierta, sirve para enfatizar su diferenciación de los alumnos tradicionales o con dedicación exclusiva. También parece claro que todavía existen diferencias entre la enseñanza a distancia tal y como se practica habitualmente y el ideal de la enseñanza tradicional. Este lugar común no revestiría ningún interés si no fuera porque en la época en que vivimos parece posible contar con los medios necesarios para esta dicotomía quedara finalmente resuelta. Hoy en día es ampliamente compartido que la enseñanza a distancia, siempre dependiente de la tecnología, acaba de entrar en una tercera fase de evolución que estará marcada por el desarrollo del potencial de la telemática.

¿Podrán utilizarse las nuevas tecnologías de la información no sólo para hacer llegar el contenido de los cursos, sino también para facilitar el acceso directo a los profesores más en vanguardia, a los más carismáticos e interesados en la investigación? ¿Mejorará la aplicación de estas nuevas tecnologías la comunicación entre los estudiantes, sus tutores y sus compañeros? ¿Les dotará de fácil acceso a importantes bases de datos así como a libros o artículos de prensa? Por último, ¿se superarán las diferencias, creándose una forma convergente e integrada de enseñanza superior que acabe con la por tanto tiempo existente dicotomía?

Y lo que es todavía más importante, ¿permitirán las nuevas tecnologías que la enseñanza a distancia cumpla en mayor profundidad con los objetivos sociales, reduciendo sus costes y aumentando sus beneficios? Este es sin duda el reto que tienen planteado las nuevas tecnologías para el próximo milenio. 


\section{REFERENCIAS BIBLIOGRÁFICAS}

1. MacArthuR, B. (1974). An interim history of the Open University. En Tunstall, J. [Ed] The Open University Opens. London: Routledge and Kegan Paul.

2. A further economic reason for state intervention lies in the difficulty individuals may meet in securing the finance required to invest in a form of capital embodied in the individual (and therefore not recoverable in the event of non-repayment). It is of course possible to advance arguments based on equity or altruism, but these still need to be judged in relation to other 'worthy' objectives. These, essentially normative judgements are beyond the scope of positive economics.

3 Schultz, T. W. (1961). Investment in Hurnan Capital En American Economic Review, 51, 1-17.

4 See, for example, the writings of Petty, Say, Von Thunen, and Fisher.

5 He went on to express the view that the additional return to the work he performed would compensate for the expense incurred in his education, with the addition of at least the profit of an equally valuable capital. Smith, A. (1776). An Inquiry into the Nature and Causes of the Wealth of Nations. Reprinted (1976) Chicago: The University of Chicago Press. [Libro 1. Cap. 10]

6. Denison, E. (1962). The Sources of Economic Growth in the United States and the Alternatives Before Us. New York: Committee for Economic Development.

7. The theory was challenged, initially on philosophical and practical grounds.

Schaffer, H. G. (1961). Investment in Human Capital: Comment. En American Economic Review, 52, 1026-1035.

Merrett, S. (1966). The Rate of Retum to Education: A Critique. En Oxford Economic Papers, 18, 289-303.

Later objections related to questions of causality (does education merely identify more able students, rather than develop their skills), and the anomalies in the earnings-education relationship (e.g. of differential returns to different categories of workers).

8 Blaug, A. (1976). The Empirical Status of Human Capital Theory: A Slightly Jaundiced View. En Journal of Economic Literature, Sept., 205-262.

9. Bååth, J. A. (1981). A Note on the Origin of Distance Education. En ICDE Bulletin, (7), 61-621.

10. Curran, C. (1997). Scale, Cost and Effectiveness in University Distance Teaching. Aldershot: Ashgate [en prensa].

11. ibid.

12. Kitchen, R. D. (1985). Men of vision: A University Challenge to Distance Education. Queensland, Australia: Division of External Studies.

University of Queensland,

13. Miller, G. (1989). Distance Education in the United States: Collaboration and Diversity. En Open Learning, 3 (4), 23-27.

14. Curran, C. (1997).

15. Commonwealth Tertiary Education Commission (1986). Review of Efficiency and Effectiveness in Higher Education. Canberra: Australian Government Publishing Service.

16. Perraton, H. (1982). The Scope of Distance Teaching. En Perraton, H. (ed) Alternative Routes to Formal Educinon: Distance Teaching for School. Baltimore and London: Johns Hopkins University.

17. Möhle, H. (1983). Higher Education and Postgraduate Studies in the GDR Organized as Distance Education. En Higher Education in Europe, 3 (VIII), 26-33.

18. And earlier still of UNISA in South Africa.

19. Open University (1993). Open University Statistics 1991. Milton Keynes: Open University.

${ }^{20}$ Two more have since been established: one (a virtual university) in Catalonia; and a second being established in Greece. There is also an 'Open University' at the University of Oulu in Finland - but there are differences between these three and the initial five open universities.

21. Data provided in response to enquiry to the UK Higher Education Statistics Agency.

22. Source: Eurostat (1997). Education Across the European Union, Statistics and Indicators. Luxembourg: CEC.

23. Source: Germany (FU) private correspondence 14/01/99 (Data includes all students on credit programmes).

Netherlands (OU) private correspondence 13/01/99 (Data includes registered students).

Portugal: (UA) Private correspondence 26/01/99.

Spain (UNED) EADTU Mini-directory 1997/98 (Data includes degree courses, continuing education, post-graduate courses and third cycle students).

United Kingdom (OU) Report of the Vice-Chancellor 1995 (Data includes undergraduate, taught and research post-graduate and associate students).

All data relates to 1995 .

24. Public expenditure per student is generally high, relative to lower levels of the education system, averaging about three times the rate of expenditure per student in primary education. The ratio varies between developed countries (from 1.78 for France to 3.36 for Japan and the 
United States, and more widely still in developing countries), see: Eicher, J. C. (1995). International Educational Expenditures. En Carnoy, M. [Ed] International of Encyclopedia of Economic of Education. 2 ${ }^{\mathrm{a}}$ Ed. Oxford: Pergamon, 443-450.

26. Ibid

27. Higher education in Germany is structured on a regional basis with individual Lander responsible for funding universities within their region. The FernUniversität is funded by the government of North-Rhine Westphalia, but enrols students throughout Germany (and in some other European countries) and is the only open university in Germany.

28. From the Higher Education Funding Council for England. Source: The Open University Report of the Vicechancellor 1997. Milton Keynes: The Open University.

29. Distance education in these countries is typically provided by one or more dual-mode universities; where funding is not separately designated for distance teaching (as is often the case), it is particularly difficult to derive even an approximate estimate of public expenditure.

30. Source:

(a) GDP: CEC (1998) European Economy Brussels: CEC Directorate General for Economic and Financial Affairs. Convergence Report 1998 N 65

(b) Expenditure on Higher Education: Derived from data in UNESCO (1998) Statistical Yearbook. Paris: UNESCO.

31. Source:

Germany (FU) private correspondence 14/01/99.

Netherlands (OU) private correspondence (Finance Department OU) 13/01/99.

Portugal: (UA) Private correspondence 25/01/99.

Spain (UNED) Private correspondence UNED 19/01/99.

United Kingdom: United Kingdom (OU) Report qf the Vice-Chancellor 1995.

32. Horlock, J

33. Wagner, L. (1972). The Economics of the Open Univesity. En Higher Education, (2), 159-183.

34 Source: EADTU (1997). Mini-directory 1997/98. EADTU: Heerlen. 1997 (except UKOU 1995/96).

35. Private expenditure is estimated to range from more than half of total national expenditure on education in India, to some 3-4\% in the Netherlands. Source: UN, National Accounts Statistics, 1986. Cited in Johnes, G. (1993). The Economics of Education. London: MacMillan Press.

35. Psacharopoulos G. (1973) Returns to Education: An Internatinal Comparison. Amsterdam: Elsevier.

36. Even though mature students in full-time education pay no fees.

37. No doubt because most study part-time and may be employed full-time. But this is clearly not the case for unemployed students, or for students managing a home.

38. Kirkwood, A. (1988). Students’ cost and hardship in !986. IET Student Research Centre. Report (12). [none]. Milton Keynes: The Open Universuy. Institute of Educational Technology.

39. Curran, C. and Fox, S. (1998). Telematic and Open and Distance Learning. Report prepared for the CEC DG XXII, Socrates Programme [Mono].

40. Psacharopoulos, G. (1973). Returns to education: an international comparison.Amsterdarn: Elsevier.

41. Source: Digest of Education Statistics US Department of Education. Cited in Eicher, J. C. (1995). Intemational Educational Expenditures. Carnoy, M. [ed] International Encyclopedia of Economic of Education. 2nd Ed. Oxford: Pergamon. 443-450.

42. See, for example, Psacharopoulos, G. (1989). Time trends of the returns of education: cross-national evidence, Economics of Education Review. 8(3): 225-239.

43. The corresponding rates for men are: $10.9 \%, 10.8 \%, 12.7 \%$ and $28.3 \%$, again for Germany, the Netherlands, the United Kingdom and Portugal, respectively. OECD (1998). Human Capital Investment: an International Comparison. Paris: OECD Centre for Educational Research and Innovation.

44. Carnoy, M. and Levin, H. M. (1975). Evaluation of Educational Media: Some Issues, Instructional Science, vol 4, $385-406$.

45. Mace, J. (1978). Mythology in the making: Is the Open University really cost-effective?, Higher Education, vol 7, $295-309$.

46. Of whom $34 \%$ had been promoted. Twenty percent said that the degree had been essential in achieving the change in employment, and $74 \%$ said it had been a help. Source: NDEC (1994). Graduate Survey [Mono]. Dublin: National Distance Education Centre, Dublin City University.

47. For example, $40 \%$ reported 'promotion to a higher grade', and $14 \%$ had achieved managerial status.

48. Woodley, A. (1988). Graduation and Beyon, Open Learning, vol 3.1, 13-17. 
49. The survey was of graduates of the Economics Department of the FernUniversität in 1986 to ascertain their perceptions of the value of their degrees in the employment market. See: Bartels, J. and Rathmore, C. S. (1989). The Value of the Distance Teaching University Degrees in the Employment market: A Study of the FernUniversität Graduates, Distance Education v.10.1, 108-118.

$50 \quad$ Bartels, J. and Arroyo...

51 The data relates to an experimental programme in Sweden which included a follow-up survey carried out five years later. See: Willen, B. (1981). Distance Education at Swedish Universities: An Evaluation of the Experimental Programme and a Follow-up Study. Uppsala Studies in Education 16. Uppsala: Almquist and Wiksell International.

$52 . \quad$ Muta and Saito $(1995) \ldots$

53. The data on graduates' earnings in 1994 were compared to the annual earnings of other workers to develop comparative age-earnings profiles; these were then used to calculate the present value of future earnings at age 22 and 41 .

54 Open University (1972). The early development of the Open Universirty: report of the Vice-chancellor, january 1969 - December 1970. Milton Keynes: The Open University.

55 Cf. Dodd, J. (1981). The Credibility of Distance Education DERG paper (1). [Mono] Milton Keynes: The Open University. Distance Education Research Group.

56. Kelly, J. (1997). Cyber campuses can't beat the real thing, Irish Times: Education and 1iving, May 13.

57 Newman, J. H. (1976). The idea of a University, Oxford: Clarendon Press.

58. Rashdall notes that word university, in medieval usage meant "...merely a number, a plurality, an aggregate of persons." And went on to note 'It is particularly important to notice that the term was generally in the Middle Ages used distinctly of the scholastic body whether of teachers or scholars, not of the place in which such a body was established, or even of its collective schools." Rashdall, H. (1936). The Universities of Europe in the Middle Ages. Oxford: Clarendon Press.

59. This common descriptor embraces an amorphous, ill-defined, but none the less real, amalgam of cultural, philosophical and spiritual dimensions of life.

60. Cf. Dowen, H. R. (1977). Investment in Learning. The Individual and Social Value of American Higher Education. San Francisco: Jossey Bass. Pascarella, E. T. and Terenzine, P. T. (1991). How College Affects Students:Findings and Insights from Twenty Years of Research. San Francisco: Jossey Bass.

61 For an interesting discussion of some differences between the system of higher education in the United States and advanced industrial countries see: Trow, M. (1991). The Exceptionalism of American Higher Education; Trow, M. and Nybom, T. [eds], The University and Society: Essays on the Social role of Research and Higher Education. Higher Education Policy Studies 12. London: Jessica Kingsley. 156-172.

62. See, for example, Teichler, U. (1993). Structures of Higher Education Systems in Europe; Gellert, C. [ed]. Higher Education in Europe. Higher Education Policy Series (16). London: Jessica Kingsley.

63. Four have been established by national governments. The FernUniversität, in keeping with practice in Germany, was established by the State of North-Rhine Westphalia.

64 Source: Open University Statistics 1991. Students, Staff and Finance. Milton Keynes: Open University. Fig.c.p. 62.

65 VanEnckevort (1986). Distance higher education, aims and experiences: country experiences. The Open universiteit of the Netherlands, vanEnckevort, G., Harry, K, Morin, P. and Schultze, H. G. [eds], Distance Higher Education and the Adult Learner. Heerlen: Open universiteit.

66 See, for example, Rumble, G. (1982). The Open University for the United Kingdom: an evaluation of an innovative experience in the democratisation of higher education. DERG Paper (6). [Mono] Milton Keynes: Open University: Distance Education Research Group.

${ }^{67}$ Woodley, A. (1981). Implementation of Higher Education Reforms. The Open Universirty of the United Kingdom. Amsterdam: The European Cultural Foundation.

68. Perry cites the case of two his "favourite students at the Open University" when he was Vice-chancellor. Neither had any school qualifications and both passed their degree in the minimum three years, never having failed an exam. Perry, W. (1986). The Adult Learner in Distance Higher Education, vanEnckevort, G., Harry, K., Morin, P. and Schultze, H. G. [eds], Distance Higher Education and the Adult Learner. Heerlen: Open universiteit.

69. Limiting the extent of drop-out is a perennial concern in distance teaching universities.

$7^{70}$ Perry, W. (1996). Distance Systems in Europe, Burgen, A. (ed), Goals and Purposes of Higher Education in the 21st Century. London: Jessica Kingsley, 62-68.

71 Bartels, J. and Rathmore, C. S. (1989). The Value of Distance Teaching University Degrees in the Employment Market: A Study of the FernUniversität Graduates, 


\section{PERFIL ACADÉMICO-PROFESIONAL DEL AUTOR}

Chris Curran se ha jubilado recientemente de su puesto de director fundador del National Distance Education Centre de Irlanda y Decano de Facultad en la Dublin City University. Economista de profesión, ha escrito e impartido extensamente sobre costes y eficacia de estrategias alternativas en las universidades a distancia. Continúa activo como investigador, tanto en Europa como en Estados Unidos. 